\title{
IX. Über Fayalit von der Insel Pantelleria.
}

\author{
Von \\ J. Soellner in Freiburg i. Br. \\ (Hierzu Tafel II, Fig. 3-6.)
}

Bei meinen Studien über den Cossyrit ${ }^{1)}$ aus dem Krater Cuddia Mida auf der Insel Pantelleria fand ich ein weiteres dunkles Mineral, das sich bei oberflächlicher Betrachtung kaum von Cossyrit abhebt. Bej näherem Zusehen unterscheidet es sich jedoch deutlich durch seine mehr gedrungene Gestalt, durch weit stärkeren Glanz seiner Flächen und sehr häufig durch seine Durchsichtigkeit in gelben bis braunen Tönen. Schon die flüchtige Prüfung des Minerals ergab den ausgesprochenen Olivincharakter desselben und die genaue krystallographische und chemische Untersuchung die Zugehörigkeit zur Fayalitreibe innerhalb der Olivingruppe.

Fayalit in guten meßbaren Krystallen gebört immer noch zu den großen Seltenheiten in der Krystallwelt, dieses Vorkommen auf Pantelleria verdient daher einiges Interesse und eine genaue Untersuchung, wie sie im folgenden gegeben ist.

Sehen wir von den künstlichen Eisenfrischschlacken $a b$, so sind es bisher nur ganz wenige Vorkommnisse von Fayalit, an denen krystallographische und chemische Untersuchungen vorgenommen werden konnten. Streng genommen sind bisher an keinem Vorkommen die krystallographischen Elemente und die chemische Zusammensetzung zugleich bestimmt worden.

Die Iddings-Gooch und Penfield'schen ${ }^{2}$ ) Untersuchungen sind an Fayaliten von verschiedenen Fundorten ausgeführt worden. Die Analyse an Krystallen von Obsidian Cliff und die krystallographischen Untersuchungen an Krystallen aus Lithophysen des massigen Obsidians, $\frac{1}{2}$ Meile

1) J. Soellner, Beiträge zur Kenntnis des Cossyrits von Pantelleria. Diese Zeitschr. 1900, 46, 318.

2) J. P. Iddings, Fayalit aus dem Yellowstone National Park. Amer. Journ. of Sc. 1885, III, 30, 58. Ref. diese Zoitschr. 1886, 11, 306. 
nördlich vom Lake of the Woods. Bei dem von Iddings und Penfield ${ }^{1)}$ beschriebenen Fayalit in Obsidian von Lipari, ebenso bei dem von $\mathrm{Scacchi}{ }^{2}$ ) und später von $\mathrm{Zambonini}{ }^{3}$ ) beschriebenen Fayalit vom Vesuv liegen nur krystallographische Messungen vor, aber keine optischen Bestimmungen und keine quantitative Analyse. Von allen anderen untersuchten natürlichen Fayalitvorkommnissen liegen nur höchstens chemische Untersuchungen oder Bestimmungen der optischen Eigenschaften ${ }^{4)}$ vor, aber keine Winkelmessungen.

Über das Vorkommen des Fayalits im Krater Cuddia Mida auf Pantelleria ist nun folgendes zu bemerken. Wie ich schon in meiner Arbeit über den Cossyrit erwähnt habe ${ }^{5}$ ), findet sich der Fayalit in gut ausgebildeten Krystallen zusammen mit Cossyrit, Quarzbipyramiden und allerhand Feldspäten usw. in einem feinen Grus, der den ebenen Kraterrand des kleinen parasitären Vulkankegels Cuddia Mida bedeckt. Der Vulkankegel Cuddia Mida, $591 \mathrm{~m}$ hoch, ist dem andesitischen Centralgebirge, der Montagna Grande, auf deren Nordwestabhange parasitisch aufgesetzt. Der Krater gehört der Epoche der Pantelleriteruptionen an. Der feine weiß und schwarz gesprenkelte Sand, der den Kraterrand bedeckt, und in dem sich der Fayalit findet, ist hauptsächlich aus der Zersetzung pantelleritischer und pantellerittrachytischer Gesteine entstanden. Das Material derselben wurde vielleicht zum Teil in zerstäubter Form ausgeschleudert und erleichterte so die Bildung des feinen Mineralgruses, der den Kraterrand bedeckt. Wie nun die mikroskopische Untersuchung der Gesteine von Cuddia Mida und ebenso von anderen Punkten der Insel lehrt, stammt der Fayalit aus den Pantelleriten wie auch aus den von Förstner als Augitandesite

1) J. P. Iddings und S. L. Penfield, Fayalit in Obsidian von Lipari. Americ. Journ. of Sc. 1890, 40, 75. Ref. diese Zeitschr. 1892, 20, 470.

2) E. Scacchi, Über die Krystallform des Neochrysoliths. Diese Zeitschr. 1889, $15,293$.

3) F. Zambonini, Mineralogia Vesuviana, Napoli 1910 , S. 236.

4) Thomson, Outlines of Min. 1836, 1, 461.

Delesse, Bull. géol. II, 10, 571 .

W. E. Hidden und J. B. Mackintosh, Mineralogische Notizen. Amer. Journ. of Sc. 1894, 41, 438. Ref. diese Zeitschr. 1896, 28, 419.

S. L. Penfield und E. H. F orbes, Über den Fayalil von Rockport, Mass., usw. Diese Zeitschr. 1896, 86, 143.

D. Lovisato, Über den verwitterten Fayalit des Granulits von Villacidro (Sardinien). Rendic. R. Acad. Lincei, Roma 1900, 9 (II), 10-13. Ref. diese Zeitschr. 1902, 35, 499.

S. Weidman, Das Vorkommen von Fayalit in gewissen Massengesteinen von Central-Wisconsin. Journ. Geol. 1904, 12,551-561. Ref. diese Zeitschr. 1907, 48, 306.

J. Soellner, l. c. S. 699. 
bezeichneten Gesteinen, die von Rosenbusch ${ }^{1}$ ) neuerdings als pantelleritische Trachyte aufgefaßt werden.

Förstner selbst scheint den Olivin resp. Fayalit in diesen Gesteinen nicht beobachtet zu haben. Rosenbusch ${ }^{2}$ ) erwähnt zuerst das Vorkommen von >Olivin * als spärlicher Gemengteil sowohl in den Pantelleriten wie auch in den $\rightarrow$ Augitandesiten a Förstner's. Meine Untersuchungen bestätigen das Vorkommen und die Verbreitung des Olivins resp. Fayalits in den Pantelleriten und in den $\gg$ Augitandesiten*. Untersucht man Schliffe der $*$ Augitandesite von der Montagna Grande, der der Krater Cuddia Mida parasitisch aufgesetzt ist, so findet man den Fayalit als spärlichen Gemengteil in fast allen Schliffen. Außer in dem *Augitandesit * von der Montagna Grande findet sich der Fayalit auch in dem stromförmigen »Augitandesite von San Gaetano an der Südwestküste von Pantelleria. In den Pantelleriten ist der Fayallit ebenfalls, wenn auch immer nur als spärlicher Gemengteil, verbreitet. Außer von Cuddia Mida habe ich ihn bis jetzt in Pantelleriten von folgenden Fundorten mikroskopisch beobachtet: Lave del Khagiar, Cala il Porticello, Monte Gelkhamar, bei Punkt $234 \mathrm{~m}$ zwischen Costa di Zichidi und Costa di Monastero, und westlich oberhalb Punta del Formaggio. Der Fayalit ist in diesen Pantelleriten und "Augitandesiten *, wenn auch nur ein spärlicher, so doch allgemein verbreiteter Gemengteil. Er tritt immer nur in einer Generation als Einsprengling auf und gebört zu den ältesten Bildungen der intratellurischen Periode. Er findet sich als EinschluB in den Anorthoklas- und ebenso in Aegirinaugiteinsprenglingen, ist also älter als diese beiden. Der Fayalit selbst ist frei von Einschlüssen.

Während der Effusivperiode dieser Gesteine wird der Fayalit offenbar bestandsunfähig und zeigt daher vielfach mehr oder weniger starke Corrosionserscheinungen, die auch an den losen Krystallen aus dem Krater Cuddia Mida sehr sohön zu beobachten sind. In seinen mikroskopischen Eigenschaften ist der Fayalit in den beiden Gesteinen vollkommen übereinstimmend, auch die Messung der verschiedenartigsten losen Krystalle hat nie eine wesentliche Differenz ergeben.

Im Gegensatz zu den Pantelleriten und Pantellerittrachyten scheint der Olivin in den Basalten (Trachydoleriten) von der Nordwestseite von Pantelleria seinen mikroskopischen Eigenschaften nach kein Fayalit zu sein, sondern ein gewöhnlicher Olivin.

Die quantitative Beteiligung des Fayalits an der Zusammensetzung des Cossyritsandes im Krater Cuddia Mida ist nur eine sehr geringe. Mehr wie die Hälfte des Sandes besteht so schon aus den hellen Gemengteilen Quarz und Feldspäten, und unter den dunklen wiegt der Cossyrit so vor,

1) H. Rosenbusch, Mikroskop. Physiogr. IV. Aufl., Stuttg. 1908, 2 (2), 926.

2) H. Rosenbusch, Mikroskop. Physiogr. II. Aufl, Stuttg. 1887, 2, 568, 686. 
daß vielleicht auf hunderte von Cossyritkryställchen ein Fayalitkryställchen kommt.

Zur Isolierung des Fayalits wurde der Sand zunăchst grob mit Acetylentetrabromid getrennt. Dadurch bekommt man sehr rasch eine vollkommene Trennung der hellen und dunklen Gemengteile. Die dunklen Mineralien wurden anfangs mit schweren Lösungen, wie Methylenjodid, Rohrbach'scher Lösung, weiter zu trennen versucht, aber mit negativem Erfolg. Beide waren schwerer als die schwersten Lösungen, Cossyrit, wie früher angegeben, 3,802, und der Fayalit, wie die spätere Bestimmung an isoliertem Material ergab, 4,24. Mit dem Elektromagneten war eine weitere Trennung der beiden Mineralien ebenfalls unmöglich, da beide ziemlich gleich gut angezogen werden. Es blieb daher nichts anderes übrig, als in der gleichen Weise, wie dies früher für den Cossyrit geschah, den Fayalit aus dem letzten schwersten Sande mit der Hand mechanisch auszusondern. Auf diesem allerdings etwas sehr mühsamen Wege wurden nach und nach mehrere Gramm Fayalitkrystalle ausgelesen, die für die krystallographische und chemische Untersuchung Verwendung fanden.

Der Habitus der Krystalle ist etwas verschiedenartig. Für gewöhnlich sind sie kurzsäulig nach der $c$-Axe mit fast vollkommen hexagonalem Habitus, entsprechend hexagonalem Prisma mit Bipyramide und Basis. Dieser scheinbar hexagonale Habitus ist bedingt durch die Formen $\{110\}$, $\{010\},\{111\},\{021\},\{001\}$. Die Formen $\{120\}$ und $\{101\}$ sind an solchen Krystallen nur sehr schmal ausgebildet. Diesen Typus zeigt Fig. 3 auf Taf. II.

Bei einem zweiten Typus sind die Krystalle ebenfalls säulig nach der $o$-Axe, aber gleichzeitig etwas tafelig nach $\{010\}$. Tafel II, Fig. 4.

Ein dritter Typus weicht von diesen beiden ziemlich stark ab. Die Krystalle desselben sind tafelig nach $\{010\}$ und gleichzeitig stark gestreckt in der Richtung der a-Axe. Siehe Tafel II, Fig. 5.

Die säulig nach der $c$-Axe ausgebildeten Krystalle des Typus I und II erreichen im allgemeinen Längen bis zu $1,25 \mathrm{~mm}$ und Dicken in den Richtungen der $a$ - und $b$ - $\Lambda$ xen von 1,1 mm. Hier und da findet man auch größere Kryställchen mit Längen bis zu 2,15 mm und Dicken bis zu $2 \mathrm{~mm}$. Die größten Krystalle sind im allgemeinen diejenigen des Typus III. Sie erreichen in der Richtung der $a$-Axe eine maximale Länge von $2,6 \mathrm{~mm}$, in der Richtung der $c$-Axe von $1 \mathrm{~mm}$ und in der Richtung der $b$-Axe von $0,7 \mathrm{~mm}$.

In ihrem Krystallhabitus weichen sonach die Fayalite von Pantelleria sehr erheblich von den meisten anderen bisher untersuchten Fayaliten ab. Die Fayalitkrystalle aus dem Yellowstone Park $\left.{ }^{1}\right)$ sind alle tafelig nach $\{100\}$

1) J. P. Iddings, Fayalit aus dem Yellowstone National Park. Amer. Journ. of Sc. 1885, 80, 58. Ref. diese Zeitschr. 1886, 11, 306. 
ausgebildet. Desgleichen die von Iddings und Penfield beschriebenen Fayalite von Lipari 1). Auch die von Lacroix aus Einschlüssen im Trachyt vom Capucin (Mont Dore) ${ }^{2}$ ) und von Santorin ${ }^{3}$ ) beschriebenen Fayalite sind nach $\{100\}$ tafelig ausgebildet. (Bei Lacroix's Aufstellung des Fayalits nach Des Cloizeaux mit $\{100\}=\{001\})$.

Nur unter den von $\left.\mathrm{Seacchi}^{4}\right)$ und $\left.\mathrm{Zambonini}{ }^{6}\right)$ beschriebenen Fayalitkrystallen vom Vesuv findet sich außer dem nach $\{100\}$ tafeligen Typus auch ein nach $\{010\}$ tafeliger Typus, entsprechend dem Typus III von Pantelleria. Im Gegensatz zu den meisten vorerwähnten Fayalitvorkommnissen zeigen die Fayalite von Pantelleria nie eine tafelige Ausbildung nach $\{100\} .\{100\}$ fehlt bei den Fayaliten von Pantelleria überhaupt vollståndig als Krystallfä̈che.

Die Farbe der frischen Krystalle ist weingelb, grünlichgelb. Die Kryställchen sind durchscheinend bis durchsichtig und besitzen auf ihren Flächen einen starken Glasglanz. Viele Krystalle sehen schwarz undurchsichtig aus. Beim Zerschlagen sind sie im Innern wie die ganz frischen, nur eine äußerste dünne Schicht ist durch Oxydation des Eisens schwarz undurchsichtig geworden. Da die Fayalite im Gestein selbst, wie die Untersuchung von Dünnschliffen zeigt, immer ganz frisch bis außen hin sind, so ist die dunkle Rinde offenbar erst bei und nach dem Auswittern aus dem Gestein entstanden. Diese dunkle Rinde ist wahrscheinlich, wie die Analyse zeigt, als ein limonitisches Zersetzungsproduct aufzufassen.

An der Oberfläche selbst der ganz frischen Kryställchen sind immer zahlreiche feine Vertiefungen, von Grundmasseneinbuchtungen herrührend, zu beobachten. Diese Corrosionserscheinungen sind ein sicheres Kennzeichen dafür, daß die losen Krystalle ursprünglich im Gestein selbst eingebettet waren und nicht in Hohlräumen saßen, wie die amerikanischen Fayalite und die von Lipari.

Da die Krystalle ringsum gut ausgebildet sind und ihre Flächen guten Glasglanz besitzen, so konnten die Krystallelemente bestimmt werden. Sieben ausgesucht gute Krystalle wurden vollständig gemessen und ergaben folgende Werte:

1) J. P. Iddings und S. L. Penfield l. c.

2) A. Lacroix, Sur la fayalite des enclaves volcaniques des trachytes du Capucin (Mont Dore). Bull. d. I. soc. franc. d. min. 1891, 14, 10. Ref. diese Zeitschr. 1894, 22, 278.

3) A. Lacroix, Sur les minéraux cristallisés, formés sous l'influence d'agents olatils, aux dépens des andésites de l'île de Théra (Santorin). Compt. rend. 1897, 125, 1189-1191. Ref. diese Zeitschr. 1899, 31, 66.

4) E. Scacchi l. c.

5) F. Zambonini l. e. 
Fayalit von Cuddia Mida, Pantelleria.

Krystallsystem: Rhombisch.

$$
a: b: c=0,46000: 1: 0,58112 \text {. }
$$

Beobachtete Formen: $b\{010\}, m\{110\}, s\{120\}, k\{021\}, c\{001\}$, $e\{111\}, d\{101\}$.

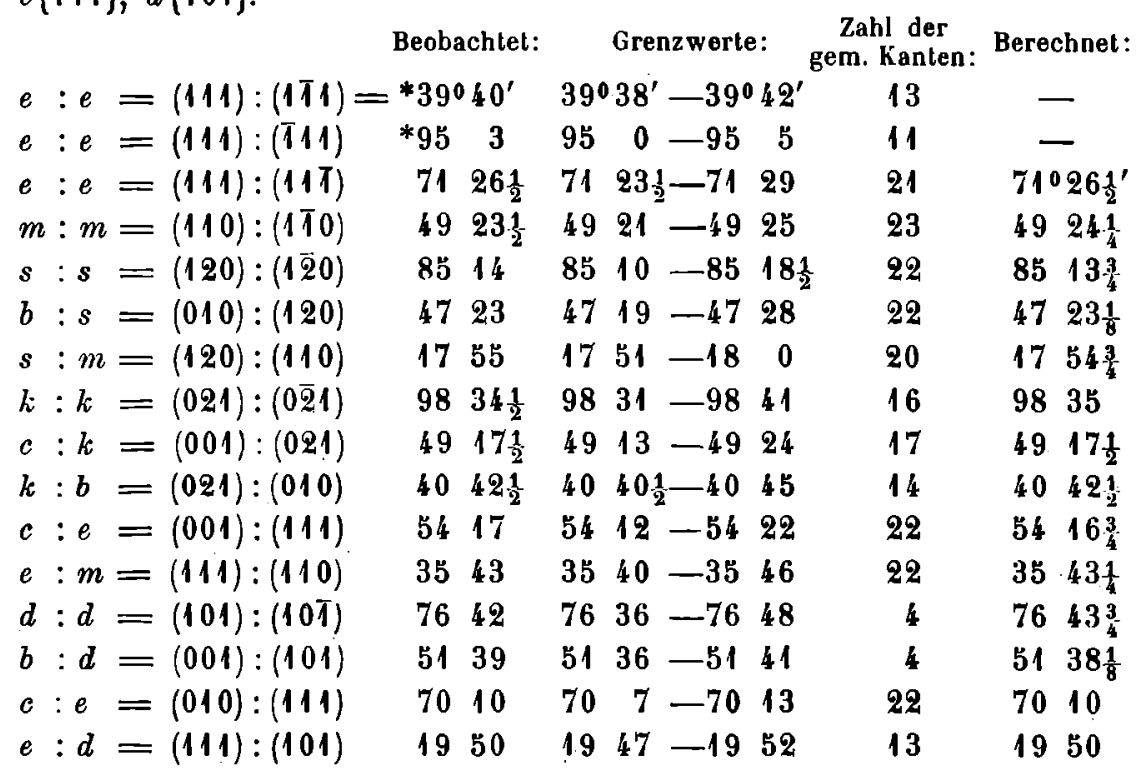

Spaltung nach $b\{010\}$ gut, weniger gut nach $c\{001\}$. Die Spaltung nach $\{010\}$ zeigt sich im Dünnschliff in zahlreichen feinen Rissen, die nach \{001\} im Vergleich dazu nur in einzelnen groben Rissen.

Die Härte ist 6,5-7.

Das specifische Gewicht wurde mit dem Pyknometer bestimmt. Die Bestimmung wurde ausgeführt mit $3 \mathrm{~g}$ des reinsten Materials, das nachher zur Analyse Verwendung fand. Das Mittel von zwei gut übereinstimmenden Bestimmungen ergab den corrigierten Wert von 4,24.

Schon von kalter conc. $\mathrm{HCl}$ wird der Fayalit unter Abscheidung von gelatinöser $\mathrm{SiO}_{2}$ vollständig zersetzt. Vor dem Lötrohre schmilzt er leicht zu einem schwarzen magnetischen Glase.

Etwa 3,5 g des reinsten mit der Hand ausgelesenen Materials wurden zur Analyse verwendet. Dieselbe wurde auf meine Veranlassung hin von Herrn Professor Dittrich in Heidelberg ausgeführt und ergab folgende Werte unter I. Unter II stehen die entsprechenden Molekularquotienten. 
Fayalit ron Cuddia Mida, Pantelleria.

Analytiker: Dittrich.

I.

\begin{tabular}{lcc}
$\mathrm{SiO}_{2}$ & $\mathbf{2 8 , 8 9 \%}$ & $\mathbf{0 , 4 7 8 3 1}$ \\
$\mathrm{TiO}_{2}$ & $\mathbf{1 , 1 9}$ & $\mathbf{0 , 0 1 4 8 5}$ \\
$\mathrm{Fe}_{2} \mathrm{O}_{3}$ & $\mathbf{5 , 0 8}$ & $\mathbf{0 , 0 3 1 7 9}$ \\
$\mathrm{FeO}$ & $\mathbf{5 6 , 0 5}$ & $\mathbf{0 , 7 7 9 7 8}$ \\
$\mathrm{MnO}$ & $\mathbf{3 , 3 9}$ & $\mathbf{0 , 0 4 7 7 5}$ \\
$\mathrm{CaO}$ & $\mathbf{0 , 7 4}$ & $\mathbf{0 , 0 1 3 1 8}$ \\
$\mathrm{MgO}$ & $\mathbf{3 , 1 1}$ & $\mathbf{0 , 0 7 7 0 6}$ \\
Alkalien & 0,42 & - \\
$\mathrm{H}_{2} \mathrm{O}$ & $\mathbf{1 , 0 7}$ & $\mathbf{0 , 0 5 9 4 0}$ \\
\cline { 2 - 3 } & $\mathbf{9 9 , 9 4 \%}$ &
\end{tabular}

Spec. Gewicht 4,24

Bemerkenswert ist der Gehalt an $\mathrm{TiO}_{2}$, die jedenfalls $\mathrm{SiO}_{2}$ isomorph vertritt. Der geringe $\mathrm{TiO}_{2}$-Gehalt bedingt aber hier anscheinend keine derartigen Abweichungen der krystallographischen Eigenschaften im Vergleich zum normalen reinen Fayalit, wie das z. B. bei den Titanolivinen im Vergleich zu den normalen Olivinen der Fall ist.

Der Gehalt an $\mathrm{Fe}_{2} \mathrm{O}_{3}$ und ebenso an $\mathrm{H}_{2} \mathrm{O}$ ist jedenfalls auf die schon obenerwähnte oberflächliche Zersetzung von manchen Fayalitkrystallen zurückzuführen. Diese Zersetzung, die sich in der Bildung einer dūnnen schwarzen Haut äußert, die viele Fayalitkryställchen umgibt, besteht in einer Oxydation des $\mathrm{FeO}$ und Aufnahme von $\mathrm{H}_{2} \mathrm{O}$, das sich jedenfalls mit $\mathrm{Fe}_{2} \mathrm{O}_{3}$ zu einem dichten Brauneisenerz verbindet. $\mathrm{He}_{2} \mathrm{O}_{3}$ und $\mathrm{H}_{2} \mathrm{O}$ der Analyse stehen untereinander fast genau im Verhältnis von 1:2.

$5,08 \% \mathrm{Fe}_{2} \mathrm{O}_{3}$ und $1,07 \% \mathrm{H}_{2} \mathrm{O}$ entsprechen einer Zusammensetzung von

$$
\begin{array}{lc}
\mathrm{Fe}_{2} \mathrm{O}_{3} & \mathbf{8 2 , 6 0 \%} \\
\mathrm{H}_{2} \mathrm{O} & \mathbf{1 7 , 4 0} \\
\cline { 2 - 2 } & \mathbf{1 0 0 , 0 0 \%}
\end{array}
$$

Für das genaue Verhältnis $1 \mathrm{Fe}_{2} \mathrm{O}_{3}, 2 \mathrm{H}_{2} \mathrm{O}$ wăren erforderlich

$$
\begin{array}{ll}
\mathrm{Fe}_{2} \mathrm{O}_{3} & 81,64 \% \\
\mathrm{H}_{2} \mathrm{O} & 18,36 \\
\cline { 2 - 2 } & 100,00 \%
\end{array}
$$

Die Werte von $\left.\mathrm{Fe}_{2} \mathrm{O}_{3}{ }^{1}\right)$ und $\mathrm{H}_{2} \mathrm{O}$ der Analyse kommen also diesem Verhältnis sehr nahe. Für die Berechnung der Fayalitformel bleibt also $\mathrm{H}_{2} \mathrm{O}$ unberücksichtigt, $\mathrm{Fe}_{2} \mathrm{O}_{3}$ muß auf $\mathrm{FeO}$ umgerechnet und mit dem

1) Geringe Mengen von $\mathrm{Fe}_{2} \mathrm{O}_{3}$ gehören wahrscheinlich zu den Alkalien, sind jedoch hier vernachlissigt worden. 
übrigen $\mathrm{FeO}$ vereinigt werden. Die Alkalien im Betrage von $0,42 \%$ sind jedenfalls auf geringe Verunreinigungen zurückzuführen, die hauptsächlich in den feinen Grundmasseneinbuchtungen sitzen. Sie kommen für die Berechnung der Fayalitformel nicht in Betracht. Geringe Mengen von $\mathrm{SiO}_{2}$ und $\mathrm{R}_{2} \mathrm{O}_{3}$ oder $R O$ sind an die Alkalien gebunden zu erachten, künnen jedoch hier vernachlässigt werden.

Nach Abzug der Alkalien und des Wassers und nach Umrechnung des Eisenoxyds in Eisenoxydul bleiht für den Fayalit folgende Zusammensetzung:

\begin{tabular}{|c|c|c|}
\hline $\mathrm{SiO}_{2}$ & $28,89 \%$ & $28,89 \%$ \\
\hline $\mathrm{TiO}_{2}$ & 1,19 & 1,19 \\
\hline $\begin{array}{c}\mathrm{FeO} \\
\text { (aus } \mathrm{Fe}_{e_{2}} \mathrm{O}_{3} \text { ) }\end{array}$ & $4, \mathbf{5} 7$ & 60,62 \\
\hline $\mathrm{FeO}$ & $56,05)$ & \\
\hline $\mathrm{MnO}$ & 3,39 & 3,39 \\
\hline $\mathrm{CaO}$ & 0,74 & 0,74 \\
\hline \multirow[t]{2}{*}{$\mathrm{MgO}$} & 3,11 & 3,11 \\
\hline & & $97,94 \%$ \\
\hline
\end{tabular}

Auf 100 umgerechnet würde die Zusammensetzung des Fayalits dann sein:

\begin{tabular}{|c|c|c|c|}
\hline $\mathrm{SiO}_{2}$ & $29,50 \%$ & 0,4884 & \multirow{2}{*}{$0,5035=1$} \\
\hline $\mathrm{TiO}_{2}$ & 1,21 & 0,0151 & \\
\hline $\mathrm{FeO}$ & 61,90 & 0,68116 & \multirow{4}{*}{$1,00197=$} \\
\hline $\mathrm{MnO}$ & 3,46 & 0,04873 & \\
\hline $\mathrm{CaO}$ & 0,76 & 0,01354 & \\
\hline \multirow[t]{2}{*}{$\mathrm{MgO}$} & 3,17 & 0,07854 & \\
\hline & $00,00 \%$ & & \\
\hline
\end{tabular}

$\mathrm{SiO}_{2}+\mathrm{TiO}_{2}$ verhalten sich also zu $\stackrel{\mathrm{II}}{\mathrm{RO}}$ ziemlich genau wie 1:2, entsprechend einem normalen Orthosilicat. Das Verhältnis von $(\mathrm{FeO}+$ $\mathrm{MnO}):(\mathrm{MgO}+\mathrm{CaO})$ ist nahezu wie $10: 1$.

$$
(\mathrm{FeO}+\mathrm{MnO}):(\mathrm{MgO}+\mathrm{CaO})=0,90989: 0,09208 \text {. }
$$

Dem Fayalit von Pantelleria kommt daher folgende Zusammensetzung zu:

$$
\begin{array}{r}
10\left(\mathrm{Fe}, \mathrm{Hin}_{2}(\mathrm{Si}, \mathrm{Ti}) \mathrm{O}_{4}\right. \\
\mathbf{1}(\mathrm{Mg}, \mathrm{Ca})_{2}(\mathrm{Si}, \mathrm{Ti}) \mathrm{O}_{4} .
\end{array}
$$

Der Fayalit von Pantelleria zeigt also ein derart starkes Vorwiegen des Fayalitmoleküls gegenüber den anderen Orthosilicatmolekülen, daß derartige Mischglieder noch ohne weiteres zum Fayalit gerechnet werden können.

Zum Vergleich mit der Analyse des Fayalits von Pantelleria stelle ich die anderen bekannten Analysen von natürlichen Fayalitvorkommnissen hier unten zusammen.

Groth . Zeitschrift f. Krystallogr. XI,IX. 


\begin{tabular}{|c|c|c|c|c|c|c|c|c|}
\hline & $\begin{array}{l}\text { Rock- } \\
\text { port 1): }\end{array}$ & $\begin{array}{l}\text { Slievec } \\
\text { a. }\end{array}$ & $\begin{array}{c}\left.\operatorname{rach}^{2}\right): \\
\text { b. }\end{array}$ & $\begin{array}{l}\text { Chey- } \\
\text { enne Mt., } \\
\text { Color. 3): }\end{array}$ & $\begin{array}{l}\text { Obsi- } \\
\text { dian, } \\
\text { Cliff }^{4} \text { ): }\end{array}$ & $\begin{array}{c}\text { Wis- } \\
\text { consin } 5):\end{array}$ & $\begin{array}{l}\text { Pantel- } \\
\text { leria: }\end{array}$ & $\begin{array}{c}\text { Sar- } \\
\text { dinien } 6)\end{array}$ \\
\hline $\mathrm{SiO}_{2}$ & 30,08 & 29,60 & 29,50 & 27,66 & 25,61 & 33,77 & 28,89 & 28,61 \\
\hline $\mathrm{TiO}_{2}$ & - & - & - & - & - & - & 1,19 & - \\
\hline $\mathrm{Fe}_{2} \mathrm{O}_{3}$ & - & - & - & - & 14,92 & 0,23 & 5,08 & 43,09 \\
\hline $\mathrm{FeO}$ & 68,12 & 68,73 & 63,54 & $\left.65,79^{7}\right)$ & 51,75 & 62,09 & 56,05 & 17,55 \\
\hline $\mathrm{MnO}$ & 0,72 & 1,78 & 5,07 & 4,17 & - & - & $\mathbf{3}, \mathbf{3 9}$ & $\mathbf{8 , 8 3}$ \\
\hline $\mathrm{MgO}$ & - & - & 0,30 & - & 1,66 & - & 3,11 & \\
\hline $\mathrm{CaO}$ & - & - & - & 0,47 & - & - & $0,74\}$ & 1,09 \\
\hline Alkalien & - & - & - & - & - & - & 0,42 & \\
\hline $\mathrm{H}_{2} \mathrm{O}$ & 0,80 & - & - & - & - & - & 1,07 & - \\
\hline Unlösl. $\mathrm{SiO}_{2}$ & $2-$ & - & - & - & 7,02 & - & - & - \\
\hline $\mathrm{Al}_{2} \mathrm{O}_{3}$ & - & - & - & - & Spur & - & - & - \\
\hline Unbestimmt & $t-$ & - & - & - & - & 3,91 & - & - \\
\hline & 99,72 & 100,11 & 98,41 & 98,09 & 100,96 & 100,00 & 99,94 & 99,17 \\
\hline
\end{tabular}

Zur Bestimmung der Brechungsindices des Fayalits von Cuddia Mida wurden mit Hilfe des Wülfing'schen Schleifapparates ${ }^{8}$ ) drei Prismen geschliffen, deren brechende Kante jeweils einer Axe parallel verläuft. Für das eine Prisma, dessen brechende Kante parallel zur $c$-Axe ist, wurde als eine Fläche eine natürliche $\{010\}$-Fläche benutzt, die andere. Fläche wurde innerhalb der verticalen Prismenzone angeschliffen. Die angeschliffene Fläche liegt bis auf eine Minute genau in der Zone. Der Winkel der brechenden Kante beträgt $23^{\circ} 40^{\prime}$. Das zweite Prisma wurde mit seiner Kante parallel zur $a$-Axe geschliffen. $\Lambda l s$ eine Fläche diente wieder eine natürliche $\{010\}$-Fläche, die zweite wurde in der Brachydomenzone angeschliffen. Die angeschliffene Fläche liegt bis auf ein bis zwei Minuten genau in dieser Zone. Der Winkel der brechenden Kante des Prismas beträgt $22023 y^{\prime}$.

An demselben angeschliffenen Krystall stand ein weiteres Prisma zur Bestimmung von $y$ zur Verfügung, gebildet von der gleichen angeschliffenen

1) Penfield und Forbes, Über den Fayalit von Rockport, Mass., I. c.

2a) Thomson l. c.

2b) Delesse l. c.

3) W. E. Hidden und J. B. Mackintosh, Mineralog. Notizen l. c.

4) J. P. Idding s, Fayalit aus den Yellowstone Park l. c.

5) S. Weidman, Das Vorkommen von Fayalit in gewissen Massengesteinen von Central-Wisconsin l. c.

6) D. Lovisato, Über den verwitterten Fayalit des Granulits von Villacidro (Sardinien) l. c.

7) Das Eisen ist in beiden Formen zugegen, wurde aber nicht besonders getrennt.

8) Es darf wohl an dieser Stelle betont werden, da $\beta$ die Anfertigung der Prismen mit Hilfe des Wülfing'schen Schleifapparates in außerordentlich schöner und eleganter Weise und sebr genau möglich war. Ohne diesen Apparat wäre es überhaupt unmöglich gewesen, aus den 1 bis $2 \mathrm{~mm}$ großen Kryställchen orientierte Prismen herzustellen. 
Fläche und von einer natürlichen $\{021\}$-Fläche. Der Winkel dieses Prismas betrug $180431_{2}^{\prime}$.

Das dritte Prisma verläuft mit seiner Kante parallel zur $b$-Axe. Da eine natürliche groß ausgebildete Fläche aus der Makrodomenzone fehlt, so mußlen bei diesem Prisma beide Flächen angeschliffen werden. Zuerst wurde das Makropinakoid angeschliffen. Eine Orientierung dieser Fläche war leicht gegeben durch $\{001\}$ und $\{010\}$. Die angeschliffene Fläche ist zu (010) $890591^{\prime}$ ' geneigt und zu (001) 890581'. Die Genauigkeit der Lage der Fläche ist also mehr wie ausreichend. Die zweite Fläche wurde in der Makrodomenzone angeschliffen und liegt genau in dieser Zone. Der Winkel der beiden angeschliffenen Flächen beträgt $28^{\circ} 38^{\prime}$. An jedem Prisma wurde mittels der Methode der Minimalablenkung immer nur je ein Brechungsindex bestimmt. Es wurde diese Methode jener anderen vorgezogen, bei welcher durch senkrechte Incidenz des Lichts auf eine Pinakoidfläche an zwei Prismen alle drei Indices bestimmt werden könnten. Auf letzlerem Wege bekommt man weit ungenauere Resultate als mit der Methode der Minimalablenkung. Zur Bestimmung des Axenwinkels wurde an natürlichen Brachypinakoidplatten der spitze Axenwinkel in Luft mit llilfe des Wülfing'schen Axenwinkelapparates gemessen und aus dem scheinbaren spitzen mit Hilfe von $\beta$ der wahre $\Lambda$ xenwinkel berechnet. Zur Controlle wurde $2 \gamma$ jeweils auch aus $\alpha, \beta, ;$ berechnet. Beide Werte zeigen gute Übereinstimmung. I) eine gewühnliche Natriumflamme zu schwache Bilder lieferte, um eine genaue Messung zı ermöglichen, so wurden die Brechungsindices und der $\Lambda$ xenwinkel für die grüne Linie und das Mittel der beiden gelben Linien im Spectrum der Quecksilberlampe von mir im hiesigen mathematisch-physikalischen Institut gemessen. Herr Prof. Königsberger stelltc mir die nütigen Hillssmittel dazu in liebenswürdigster Weise zur Verfügung. Die grüne Linie im Quecksilberspectrum entspricht einer Vellenlänge von $0,5461 \mu$, das Mittel der beiden gelben Linien einer Wellenlänge von $0,5779: 3, a$. Zum Vergleich sei daran erinnert, daß Thalliumlicht einer Wellenlänge von $0,5349, "$ und Natriumlicht von $0,5893 \mu$ entspricht.

Brechungsindices und Axenwinkel von Fayalit von Cuddia Mida, Pantelleria, für die grüne Linie $(0,5461, \iota)$ und das Mitlıl der beiden gelben Linien $(0,57795 \mu)$ im Spectrum der Quecksilberlampe:

$$
\begin{aligned}
\gamma \text { grün }(H g) & =1,85404 & \gamma \text { gellb }(I g) & =1,84625 \\
\beta \text { grün }(H g) & =1,84304 & \beta \text { gell } ! I I g) & =1,83896 \\
\alpha \text { grün }(I I g) & =1,80867 & \alpha \text { gelb }(I I g) & =1,80449 \\
(\gamma-\alpha) \text { grün }(H g) & =0,04237 & (\gamma-\alpha) \text { gelb }(I I g) & =0,04483 \\
(\gamma-\beta) \text { grün }(H g) & =0,00800 & (\gamma-\cdots) \text { gelb } I I g) & =0,00799 \\
(\beta-\alpha) \text { grün }(H g) & =0,03437 & (\beta-\alpha) \text { gelb } I I g) & =0.0338 .1
\end{aligned}
$$




$$
\begin{gathered}
\text { ( } \gamma \text { grün }-\gamma \text { gelb) } H g=0,00479 \\
(\beta \text { grün }-\beta \text { gelb) } H g=0,00478 \\
(\alpha \text { grün }-\alpha \text { gelb) } H g=0,00425 . \\
2 E \text { grün } H g=103^{0} 43^{\prime} 15^{\prime \prime} \\
2 V \text { grün } H g=503115 \text { (aus } 2 E \text { und } \beta \text { ) } \\
2 V \text { grün } H g=5042 \quad \text { (aus } \alpha, \beta, \gamma \text { ). } \\
2 E \text { gelb } H g=104041^{\prime} 15^{\prime \prime} \\
2 V \text { gelb } H g=51115 \text { (aus } 2 E \text { und } \beta \text { ) } \\
2 V \text { gelb } H g=51230 \text { (aus } \alpha, \beta, \gamma \text { ). }
\end{gathered}
$$

Die optische Orientierung des Fayalits von Pantelleria ist folgende:

$$
\begin{aligned}
& a=\mathfrak{c} \\
& b=\mathfrak{a} \\
& c=\mathfrak{b} .
\end{aligned}
$$

Die Ebene der optischen Axen ist $\|\{001\}$. Auf $\{010\}$ trilt die spitze Bisectrix, auf $\{100\}$ die stumpfe senkrecht aus. a ist spitze Bisectrix, der optische Charakter daher negativ. Die Dispersion der optischen Axen ist $0>n$.

Fig. 6 auf Taf. II gibt eine Übersicht der optischen Orientierung in bezug auf die Krystallformen.

Die Lichtbrechung und Doppelbrechung sind, wie sich aus den angeführten Werten ergibt, sehr hoch. Die Polarisationsfarben auf den drei Hauptflächen, den Pinakoiden, sind sehr verschieden. Auf $\{010\}$ ist entsprechend dem sehr niederen Wert $(\gamma-\beta)$ zum Beispiel die Polarisationsfarbe eines ca. $0,03 \mathrm{~mm}$ dicken Schliffes nur ,grünlichweiß a bis fast reinweiß « in der I. Ordnung, infolge der Eigenfarbe des Minerals jedoch scheinbar gelblich. Auf $\{100\}$ bei gleicher Dicke des Schliffes infolge des betrüchtlich höheren Wertes $(\beta-\alpha)$ Rot II. Ordnung. Am hüchsten ist die Farbe auf $\{001\}(\gamma-\alpha)$, bei gleicher Dicke des Schliffes $(0,03 \mathrm{~mm})$ grünlichblau bis meergrün in der III. Ordnung.

Der Pleochroïsmus ist in dickeren Platten sehr deutlich. Er wurde an ca. $0,25 \mathrm{~mm}$ dicken Platten bestimmt. $\Lambda$ n solchen ist $\mathfrak{b}$ orangegelb, $\mathfrak{a}=\mathfrak{c}$ grünlichgelb. Mit zunehmender Dünne der Platten wird der Pleochroïsmus selbstverständlich stark abgeschwächt. Aber selbst im Gesteinsdünnschliff ist der Pleochroïsmus des Fayalits noch deutlich wahrnehmbar. Während die gewöhnlichen Olivine im Dünnschliff vollkommen farblos durchsichtig sind und keine wahrnehmbaren Absorptionsunterschiede mehr zeigen, besitzt im Gegensatz dazu der Fayalit selbst in normalen Gesteinsdünnschliffen von ca. $0,03 \mathrm{~mm}$ Dicke noch eine charakteristische blaßgelbliche Eigenfarbe und deutlich wahrnehmbare Absorptionsunterschiede. Die Absorptionsfarbe für $c=\mathfrak{b}$ ist im Dünnschliff noch deutlich gelblich, für 
$a=\mathfrak{c}$ und $b=\mathfrak{a}$ blaßgelblich mit einem Stich in's Grünliche. Die deut_ liche Eigenfärbung des frischen Fayalits und sein Pleochroïsmus sind selbst im Gesteinsdünnschliff noch ein wichtiges diagnostisches Unterscheidungsmerkmal für den Fayalit gegenüber den gewöhnlichen Olivinen. $E s$ ist das einzig sichere Merkmal, um auf rein mikroskopischem Wege (abgesehen von einer etwaigen Messung des Axenwinkels), ohne Zuhilfenahme einer chemischen Analyse den Fayalit sofort von den übrigen olivinen zu unterscheiden.

Meines Wissens ist auf dieses wichtige mikroskopische Unterscheidungsmerkmal für den Fayalit noch nirgends hingewiesen. Es kommt das wohl daher, daß unzweifelhafter Fayalit eingewachsen im Gestein bisher noch nicht bekannt und untersucht war. Die optischen Angaben über Fayalit bei anderen Autoren beziehen sich immer nur auf die Untersuchung von losem Material, oder auf Krystalle, die in Hohlräumen saßen, aber nicht auf eingewachsene Krystalle.

Vergleicht man die krystallographischen Constanten des Fayalits von Pantelleria mit denen anderer Fayalite, soweit von solchen Messungen vorliegen, so ergibt sich folgendes. Das Axenverhältnis des Fayalits von Pantelleria kommt sehr nahe dem des Fayalits vom Yellowstone Park, von Penfield 1) aufgestellt, und ebenso dem des Fayalits vom Vesuv, das von Zambonini ${ }^{2}$ ) angegeben wird. Die Werte sind:

$$
\begin{array}{clcllc} 
& & a & : b: c & & F e \\
\text { Fayalit, Yellowstone Park } & 0,4584 & : 1: 0,5793 & \text { (Penfield) } & 65,49 \% \\
- & \text { Vesuv } & 0,4594 & : 1: 0,5806 & \text { (Zambonini) } & - \\
- & \text { Pantelleria } & 0,46000: 1: 0,58112 & \text { (Soellner) } & 61,90
\end{array}
$$

Die Differenzen sind bedingt durch die wechselnde chemische Zusammensetzung. Von dem Fayalit vom Vesuv liegt leider keine Analyse vor es scheint aber, als sei mit der Abnahme des $\mathrm{FeO}$-Gehaltes ein Wachsen der Längen der Axen $a$ und $c$ verknüpft. Grenzwerte würden sein:

$$
\begin{array}{lc}
a: b: c \\
\text { Fayalit, Yellowstone Park } & 0,4584: 1: 0,5793 \\
\text { Forsterit, Vesuv } & 0,4666: 1: 0,5868
\end{array}
$$

Ob diese Gesetzmäßigkeit für die ganze Olivinreihe vom Fayalit bis zum Forsterit zutrifft, ist zur Zeit noch fraglich, da zum Beispiel der Hyalosiderit von der Limburg am Kaiserstuhl mit 30\% $\mathrm{FeO}$ und $a: b: c=$ $0,46815: 1: 0,58996^{3}$ ) aus der Reihe herausfällt.

1) Penfield l. c.

2) Zambonini l. c. 237.

3) M. Bauer, Beiträge zur Mineralogie. Neues Jahrb. f. Min. usw. 1887, 1, 19. 
Bezüglich der optischen Eigenschaften, Lichtbrechung, Doppelbrechung, Größe des Axenwinkels ist schon von Penfield und Forbes ${ }^{1)}$ eine Gesetzmäßigkeit und Abhängigleit der betreflenden Werte vom $\mathrm{FeO}$-Gehalt nachgewiesen worden. Danach ist mit zunehmendem $\mathrm{FeO}$-Gehalt bei den Olivinen ein Zunehmen der Lichtbrechung, eine Zunahme der Stärke der Doppelbrechung und eine $\Lambda$ bnahme des Winkels der optischen Axen auf $\{0.10\}$ um a verbunden. Reiner Fayalit zeigt die höchste Lichtbrechung, die stärkste Doppelbrechung und den kleinsten Axenwinkel un $\mathfrak{a}$ auf $\{010\}$ innerhalb der Forsterit-Olivin-Fayalitreihe.

Von annähernd reinem Fayalit liegen Bestimmungen der optischen Constanten vor nur an Fayalit von Rockport, Mass., durch Penfield und Forbes ${ }^{2}$ ). Entsprechend seinem geringeren Gehalt an $\mathrm{FeO}$ besitzt der Fayalit von Pantelleria auch etwas geringere Werte bezüglich seiner Licht- und Doppelbrechung und einen etwas größeren $A$ xenwinkel auf $\{010\}$ als der Fayalit von Rockport. Die Gegenüberstellung der entsprechenden Werte zeigt dies ohne weiteres ${ }^{3}$ ).

Fayalit: $\quad F_{e} O \quad a$ gelb: is gelb: $\gamma$ gelb: $(\gamma-a)$ gelb: $2 V$ gelb:

Rockport $\quad 68,12 \quad 1,8236 \quad 1,8642 \quad 1,8736 \quad 0,050 \quad 49^{\circ} 50^{\prime} \quad 0^{\prime \prime}$

Pantelleria $\quad 61,90 \quad 1,80442 \quad 1,83826 \quad 1,84625 \quad 0,04183 \quad 34 \quad 1 \quad 15$

Der Fayalit von Pantelleria fügt sich in die von Penfield und Forbes $\left.{ }^{4}\right)$ aufgestellte Tabelle bezüglich dieser Gesetzmäßigkeiten vollständig ein. Dieselbe sei mit Ergänzung durch den Fayalit von Pantelleria hier wiedergegeben.

\begin{tabular}{|c|c|c|c|c|}
\hline & & \multicolumn{3}{|c|}{$2 V$ gelb } \\
\hline Mineral: & Fundorl: & $\mathrm{FeO}_{\mathrm{C}} \mathrm{O}$ & gemessen über a & $\beta$ gell : \\
\hline Fayalit & IRockport & 68,1 & $49^{0} 50^{\prime}$ & 1,864 \\
\hline × & Pantelleria & 61,9 & 511 & 1,838 \\
\hline Hortonolith & Monroe & 47,3 & 6924 & 1,791 \\
\hline Olivin & Auvergne & 13,0 & 8936 & 1,692 \\
\hline$\gg$ & Vesuv & 12,6 & $89 \quad 42$ & - \\
\hline , & Hawaii & 10,3 & 912 & - \\
\hline 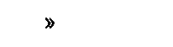 & Ägypten & 9,2 & $91 \quad 19$ & 1,678 \\
\hline$»$ & Neu-Mexico & 8,6 & $912 k$ & - \\
\hline
\end{tabular}

1) Penfield und liorbes l. c. 143 u. [.

2) Penfield und Forbes I. c. 1/4.

3) Bei der Vergleichung der Werte ist allerdings'su berücksichtigen, daß die Werte lür gelb nicht für die genau gleiche Wellenlänge gelten. Die Messungen von Penfield und Forbcs beziehen sich auf "Gelb» ohne nähere Angabe, vermutlich auf $N a$-Licht. Meine Messungen auf das Gelb im $I g$-Spectrum. Für $N a$-Licht würden die Brechungsindices und die Doppelbrechung des Fayalits von Pantelleria etwas niedriger und der Axenwinkel etwas größer sein.

i) Penfield und Forbes l. c. 147. 


\begin{tabular}{|c|c|c|c|c|}
\hline \multirow{3}{*}{$\begin{array}{l}\text { Mineral: } \\
\text { Olivin }\end{array}$} & \multirow{3}{*}{$\begin{array}{l}\text { Fundort: } \\
\text { Unbekannt }\end{array}$} & \multicolumn{3}{|c|}{$2 V$ gelb } \\
\hline & & $\mathrm{FeO} \%$ & gemessen über $a$ & $\beta$ gelb: \\
\hline & & - & $92^{0} 14^{\prime}$ & 1,678 \\
\hline , & Ostindien & - & 9245 & 1,670 \\
\hline Forsterit & Vesuv & - & 9350 & 1,657 \\
\hline
\end{tabular}

Zusammenstellung der Eigenschaften des Fayalits von Cuddia Mida, Pantelleria.

Fayalit von Cuddia Mida, Pantelleria.

$$
\begin{array}{r}
10(\mathrm{Fe}, \mathrm{Mn})_{2}\left(\mathrm{Si}, \mathrm{Ni}_{i}\right) \mathrm{O}_{4}, \\
1(\mathrm{Mg}, \mathrm{Ca})_{2}\left(\mathrm{Si}, \mathrm{Ti}_{i}\right) \mathrm{O}_{4} .
\end{array}
$$

Krystallsystem: Rhombisch.

$$
a: b: c=0,46000: 1: 0,58112 \text {. }
$$

Auftretende Formen: $b\{010\}, m\{110\}, s\{120\}, k\{021\}, c\{001\}$, $e\{111\}, d\{101\}$.

Winkeltabelle: siehe S. 143.

Habitus der Krystalle: entweder säulig nach der $c$-Axe mit scheinbar hexagonalem Habitus, oder tafelig nach $\{010\}$, oder säulig nach der $a-$ Axe und gleichzeitig tafelig nach $\{010\}$.

Spaltbarkeit: nach $\{010\}$ gut, ziemlich gut nach $\{001\}$.

Spec. Gewicht: 4,24.

Härte: 6,5-7.

Farbe: weingelb bis gelbgrün, durchsichtig bis durchscheinend. Durch oberflächliche Oxydation häufig von einer dünnen schwarzen Haut überzogen, im Innern aber frisch.

Glanz: starker Glasglanz.

Lichtbrechung: sehr hobe Lichtbrechung

$\alpha$ grün $(H g) 1,80867 \quad \beta$ grün $(H g)$ 1,84304 $\gamma$ grün $(H g) 1,85104$

$\alpha$ gelb $(H g) 1,80442 \quad \beta$ gelb $(H g) 1,83826 \quad \gamma$ gelb $(H g) 1,84625$.

Doppelbrechung: starke Doppelbrechung $(\gamma-\alpha)$ grün $(H g)$ 0,04237, $(\gamma-\alpha)$ gelb $(H g) 0,04183, \varrho>v . \quad 2 V$ grün $(H g) 50^{0} 31^{\prime} 15^{\prime \prime}, 2 V$ gelb $(H g)$ $5101^{\prime} 15$ ". Optischer Charakter negativ.

Optische Orientierung: $a=\mathfrak{c}, b=\mathfrak{a}, c=\mathfrak{b}$.

Pleochroïsmus: in Platten von ca. $0,25 \mathrm{~mm}$ Dicke $\mathfrak{b}$ orangegelb, $\mathfrak{a}=\mathfrak{c}$ grünlichgelb; im Dünnschliff $\mathfrak{b}$ gelblich, $\mathfrak{a}=\mathfrak{c}$ blaßgelblich mit einem Stich in's Grünliche.

Reactionen: von $\mathrm{HCl}$ unter Abscheidung gelatinöser $\mathrm{SiO}_{2}$ zersetzt, vor dem Lötrohre zu schwarzem magnetischem Glase schmelzend. 